\title{
Direct comparison of the potency of human mesenchymal stem cells derived from amnion tissue, bone marrow and adipose tissue at inducing dermal fibroblast responses to cutaneous wounds
}

\author{
XIAOYU LIU ${ }^{1}$, ZHE WANG $^{1}$, RUI WANG ${ }^{1}$, FENG ZHAO $^{1}$, PING SHI $^{2}$, YIDE JIANG ${ }^{1}$ and XINING PANG ${ }^{1}$ \\ ${ }^{1}$ Key Laboratory of Cell Biology, Ministry of Public Health of China, Department of Stem Cells \\ and Regenerative Medicine, ${ }^{2}$ Department of General Practice, The First Affiliated Hospital, \\ China Medical University, Shenyang, Liaoning 110001, P.R. China
}

Received September 12, 2012; Accepted November 9, 2012

DOI: 10.3892/ijmm.2012.1199

\begin{abstract}
Although transplantation of human mesenchymal stem cells (MSCs) derived from amnion (hAMSCs), bone marrow (hBMSCs) and adipose tissues (hADSCs) has been shown to aid in the repair of cutaneous wounds in mouse models, little information is available regarding the relative efficacy of MSCs from different sources. In this study, we compared their therapeutic potentials by transplanting equal numbers of hAMSCs, hBMSCs or hADSCs in a mouse model of cutaneous wounds. The results suggested that an hADSC injection has the most pronounced effect on wound closure. Histological evaluation showed enhanced re-epithelialization in the hADSC group compared with the hBMSC and hAMSC groups. Although there was a slight improvement in wound healing in the hAMSC and hBMSC groups, the differences between the groups were statistically insignificant. In a transwell coculture model, wound healing migration and transwell
\end{abstract}

Correspondence to: Professor Xining Pang, Key Laboratory of Cell Biology, Ministry of Public Health of China, Department of Stem Cells and Regenerative Medicine, China Medical University, Shenyang, Liaoning 110001, P.R. China

E-mail: pxining@yahoo.com

Professor Ping Shi, Department of General Practice, The First Affiliated Hospital, China Medical University, Shenyang, Liaoning 110001, P.R. China

E-mail: shiping57428@yahoo.com

Abbreviations: MSCs, mesenchymal stem cells; hAMSCs, human mesenchymal stem cells derived from amnion; hBMSCs, human mesenchymal stem cells derived from bone marrow; hADSCs, human mesenchymal stem cells derived from adipose tissue; hDF, human dermal fibroblast; ECM, extracellular matrix; SPIONs, superparamagnetic iron oxide nanoparticles; PFA, paraformaldehyde

Key words: human mesenchymal stem cells derived from amnion, human mesenchymal stem cells derived from bone marrow, human mesenchymal stem cells derived from adipose tissue, cutaneous wounds, proliferation, migration, cytokines, extracellular matrix migration assays showed that hADSCs were superior to hAMSCs and hBMSCs at promoting human dermal fibroblast (hDF) migration. However, there was no significant difference in fibroblast proliferation between the hAMSC, hBMSC and hADSC groups, as measured by WST assay. Our results also indicated that hDFs cocultured with hADSCs for $48 \mathrm{~h}$ significantly upregulated their mRNA expression of the cytokine vascular endothelial growth factor, basic fibroblast growth factor, keratinocyte growth factor and transforming growth factor- $\beta$ and increased the mRNA and protein levels of type I collagen. Collectively, these data suggest that hADSCs are a potential source of MSCs for therapeutic healing in cutaneous wounds in terms of efficacy, accessibility and availability.

\section{Introduction}

Cutaneous wounds are caused by surgical and accidental lacerations, burns, cuts, abrasions, and diabetic foot ulcers, among other causes (1). If not repaired, the skin may lose its barrier function and expose the underlying tissues to microbial infections and mechanical damage. In the biological process of skin wound repair, dermal fibroblasts interact with the surrounding cells and produce various cytokines, adhesive molecules, glycoproteins and extracellular matrix (ECM). These cell-to-cell, cell-to-cytokine and synthetic interdependencies allow skin fibroblasts to contribute to the fibroblast-keratinocyte-endothelium complex to repair wounds and maintain the integrity of the skin (2). Although there has recently been much interest in the use of growth factors to treat cutaneous wounds, the effects have not been very successful. Cell-based therapies have become a promising strategy for the treatment of cutaneous wounds (3).

Among numerous progenitor cell types reported to have potential in the development of tissue engineering products, mesenchymal stem cells (MSCs) have been proposed as a prominent candidate (4). MSCs have been successfully isolated from various tissues including bone marrow, peripheral blood, amnion, adipose tissues and many others, and these cells can differentiate into osteoblasts, chondrocytes, neurons, skeletal muscle cells, endothelial cells and vascular smooth muscle cells (5-9). MSCs have been considered ideal for cell therapy 
due to their simple isolation techniques, easy expandability, low immunogenicity and pluripotency (10-14).

Current studies have demonstrated that MSCs secrete soluble factors that induce dermal fibroblast proliferation and migration to enhance wound repair $(10,15)$. This enhanced wound repair has been observed in acute wounds in both non-diabetic and diabetic mouse models as well as in acute and chronic wounds in humans (16-20). The purpose of this study was to compare the therapeutic potencies of human MSCs derived from amnion (hAMSCs), bone marrow (hBMSCs) and adipose tissue (hADSCs) in inducing dermal fibroblast responses to cutaneous wounds. Our data indicated that hADSCs have superior potential in terms of accelerating wound healing compared with hAMSCs and hBMSCs.

\section{Materials and methods}

Cells and cell culture. hAMSCs. Amnion samples were obtained from Shengjing Hospital of China Medical University (Shenyang, China) and all samples were obtained with patient informed consent. Freshly isolated amniotic membranes were washed with phosphate-buffered saline (PBS) to remove the blood and then immersed in an antibiotic solution for $20 \mathrm{~min}$. Then, the amnions were cut into $25 \mathrm{~cm}^{2}$ pieces, transferred into a $0.25 \%$ trypsin solution $(0.25 \%$ trypsin; Gibco) and incubated at $37^{\circ} \mathrm{C}$ for $1 \mathrm{~h}$. The digested tissue was centrifuged, and the supernatant was discarded; this process was repeated three times. Culture medium was added after the last digestion to neutralize the digestive enzymes and end the digestion. The amniotic membrane pieces were then incubated at $37^{\circ} \mathrm{C}$ in PBS containing $1 \mathrm{mg} / \mathrm{ml}$ collagenase (Sigma-Aldrich, St. Louis, MO, USA) and $0.1 \mathrm{mg} / \mathrm{ml}$ DNase (Takara Bio, Inc., Japan) for $1 \mathrm{~h}$. An equal amount of culture medium was then added to end digestion. Following this digestion, the resulting cell suspension was filtered through a cell sieve to collect the cells, which were plated into a culture flask (designated as $\mathrm{P}_{0}$ ) in Dulbecco's modified Eagle's medium (DMEM; Gibco) containing 10\% fetal bovine serum (FBS; Hyclone), $100 \mathrm{U} / \mathrm{ml}$ penicillin and $100 \mu \mathrm{g} / \mathrm{ml}$ streptomycin. The flasks were transferred into a $37^{\circ} \mathrm{C}, 5 \% \mathrm{CO}_{2}$ saturated humidity incubator to continue culturing. The culture medium was changed every two or three days. The cells were passaged at a 1:3 ratio when they reached $80-90 \%$ confluency. For the experiment, hAMSCs were used at passages 3-6.

hBMSCs. Bone marrow samples were obtained from Shengjing Hospital of China Medical University (Shenyang, China) and all samples were obtained with patient informed consent. Approximately 10 to $30 \mathrm{ml}$ of bone marrow aspirate was collected in a syringe containing 10,000 IU heparin to prevent coagulation. Bone marrow aspirates were mixed with DMEM containing $10 \%$ FBS and centrifuged at 1,200 x g for $5 \mathrm{~min}$ to obtain a cell pellet. These cells were seeded into $25 \mathrm{~cm}^{2}$ tissue culture flasks and cultured in DMEM containing $10 \% \mathrm{FBS}, 100 \mathrm{U} / \mathrm{ml}$ penicillin, and $100 \mu \mathrm{g} / \mathrm{ml}$ streptomycin (4). After 48 h, non-adherent cells were removed, and fresh medium was added. The medium was changed every two or three days. The adherent spindle-shaped cells were further propagated for three passages. For the experiment, hBMSCs were used at passages 3-6.
hADSCs. Adipose samples were obtained from Shengjing Hospital of China Medical University and all samples were obtained with patient informed consent. To isolate hADSCs, adipose tissues were washed with PBS and digested with $0.075 \%$ type I collagenase under gentle agitation at $37^{\circ} \mathrm{C}$ for 30 min (4). Enzyme activity was neutralized with DMEM containing $10 \%$ FBS, and the suspensions were centrifuged at $300 \mathrm{x}$ g for $10 \mathrm{~min}$ to obtain a pellet. The pellet was filtered with a $70 \mu \mathrm{m}$ nylon mesh filter, suspended in DMEM containing $10 \% \mathrm{FBS}, 100 \mathrm{U} / \mathrm{ml}$ penicillin and $100 \mu \mathrm{g} / \mathrm{ml}$ streptomycin. The cultures were maintained at $37^{\circ} \mathrm{C}$ in a humidified atmosphere containing $5 \% \mathrm{CO}_{2}$. After $48 \mathrm{~h}$, the non-adherent cells were removed, and fresh medium was added to the cells. When the monolayer of adherent cells reached confluence, they were trypsinized, resuspended in DMEM containing 10\% FBS. For the experiment, hADSCs were used at passages 3-6.

Culture of dermal fibroblasts. Skin samples were obtained from Shengjing Hospital of China Medical University and all samples were obtained with patient informed consent. Fresh healthy skin tissue was de-epithelialized and minced into $2 \mathrm{~mm}$ pieces (3). These pieces were spread on the surface of a $100 \mathrm{~mm}$ tissue culture plate that was precoated with $3 \mathrm{ml}$ of DMEM containing $10 \% \mathrm{FBS}, 100 \mathrm{U} / \mathrm{ml}$ penicillin and $100 \mu \mathrm{g} / \mathrm{ml}$ streptomycin. The plates were incubated at $37^{\circ} \mathrm{C}$ for $4 \mathrm{~h}$ to facilitate tissue adhesion. Following this incubation, $12 \mathrm{ml}$ of DMEM containing $10 \%$ FBS was added, and the plates were returned to the incubator at $37^{\circ} \mathrm{C}$ with $5 \% \mathrm{CO}_{2}$. When the fibroblasts reached $80-90 \%$ confluency, the cells were passaged for expansion. For the experiments, human dermal fibroblast (hDF) cells were used at passages 3-6.

Flow cytometric characterization of MSCs. Flow cytometry was used to detect stem cell-related cell surface markers on the cultured cells. MSCs (hAMSCs, hBMSCs or hADSCs) were cultured in their corresponding growth medium for $72 \mathrm{~h}$ prior to analysis. The cells were trypsinized with $0.05 \%$ trypsin-EDTA, collected in regular mesenchymal media and centrifuged for $5 \mathrm{~min}$ at $800 \mathrm{x} \mathrm{g}$ at $4^{\circ} \mathrm{C}$. Then, the cells were resuspended in PBS containing $1 \%$ bovine serum albumin at $10^{6}$ cells $/ \mathrm{ml}$ and incubated with monoclonal PE-conjugated antibodies for CD45, CD34, CD14 and HLA-DR, APC-conjugated antibodies for CD45, and FITC-conjugated antibodies for CD90 and CD105 (all were from Becton-Dickinson, USA) for $45 \mathrm{~min}$ on ice. The cells were subsequently washed with PBS, fixed with $1 \%$ formaldehyde, and analyzed by flow cytometry. A minimum of 10,000 cell events per assay were acquired on a FACSCalibur flow cytometer.

In vitro functional assays to determine the differentiation capacity of cultured MSCs. MSCs (hAMSCs, hBMSCs or hADSCs) between passages 3 and 6 were tested for their ability to differentiate into osteocytes, chondrocytes and adipocytes (21). To induce osteogenic differentiation, cells were cultured for three weeks in DMEM with $10 \% \mathrm{FBS}$, $0.1 \mu \mathrm{M}$ dexamethasone (Amresco), $50 \mu \mathrm{g} / \mathrm{ml}$ ascorbic acid and $10 \mathrm{mM}$ glycerophosphate (were from Sigma-Aldrich), and the medium was changed every three days. After 21 days, the cells were analyzed by alkaline phosphatase staining. To induce adipogenic differentiation, cells were cultured for two weeks in DMEM with $10 \%$ FBS, $1 \mu \mathrm{M}$ dexamethasone, $0.5 \mu \mathrm{M}$ 
isobutylmethylxanthine, $60 \mu \mathrm{M}$ indomethacin and $170 \mu \mathrm{M}$ insulin (Sigma-Aldrich), and the medium was changed every two days. After 14 days, the cells were analyzed by Oil Red O staining (Sangon Biotech). To induce chondrogenic differentiation, cells were cultured for three weeks in DMEM with $10 \%$ FBS, $50 \mu \mathrm{g} / \mathrm{ml}$ ascorbic acid, $100 \mu \mathrm{g} / \mathrm{ml}$ sodium pyruvate (Sigma-Aldrich), $0.1 \mu \mathrm{M}$ dexamethasone and $10 \mathrm{ng} / \mathrm{ml}$ transforming growth factor (TGF)- $\beta 1$ (PeproTech), and the medium was changed every three days. After 21 days, the cells were analyzed by Alcian blue (Sangon Biotech) staining.

Cell labeling. PEI-coated $\mathrm{Fe}_{3} \mathrm{O}_{4}$ nanoparticles are superparamagnetic iron oxide nanoparticles (SPIONs) $(19,22-24)$. To track MSCs in vivo, cells were grown to confluence in cell culture dishes and on culture glass slides and treated with SPIONs at a final concentration of 20-30 $\mu \mathrm{g} / \mathrm{ml}$ (MSC culture medium) for $24 \mathrm{~h}$. To determine the presence of intracellular SPION particles, slide-cultured cells were fixed with $10 \%$ neutral-buffered formalin for $30 \mathrm{~min}$, rinsed with PBS and then stained with Prussian blue. The slide was immersed in a solution consisting of equal parts of $20 \% \mathrm{HCl}$ and $10 \%$ ferrocyanide for $20 \mathrm{~min}$ and then rinsed in tap water. Counterstaining with nuclear fast red was performed for $5 \mathrm{~min}$.

Wound healing model. All animal procedures were reviewed and approved by the China Medical University Review Committee. Mice (8-week-old female, body weight 20-23 g) were obtained from the Lab Animal Center of China Medical University. The animals were randomly divided into four groups ( 9 mice/group). Following hair removal from the dorsal surface, the mice were anesthetized with sodium pentobarbital $(0.5 \mathrm{mg} / \mathrm{g})$, and a $10-\mathrm{mm}$ full-thickness excisional skin wound was made on the back of each mouse $(25,26)$. MSCs were incubated with SPIONs for in vivo cell tracking using Prussian blue staining. Each wound received 1 million labeled cells (injected hAMSCs, hBMSCs or hADSCs as the experimental groups or injected PBS as the control) via 4 injections of $0.25 \times 10^{6}$ cells in $60 \mu \mathrm{l}$ of PBS, injected intradermally around the wound at four different injection sites, and dressed with fresh human amnion as an antimicrobial agent. All mice were housed individually.

Wound analysis and histological assessment. Digital images of the wounds were captured at Days 0,3, 7, 10 and 14 with a 10-megapixel digital camera (Olympus), and an image analyzer (Image-Pro Plus 6.0) was used to take wound-size measurements. Wound healing was quantified by calculating the remaining wound area for each group at $0,3,7,10$ and 14 days after grafting. The percentage of wound closure was calculated as follows: (area of original wound - area of actual wound)/area of original wound x100 (15). Mice were sacrificed 7,14 and 21 days after grafting for histological assessment. The harvested wound areas, including a border of normal tissue, were immediately fixed in $10 \%$ neutral-buffered formalin. The specimens were embedded in paraffin, sectioned, and stained with hematoxylin and eosin (H\&E) to determine the quality of wound healing as measured by the characteristics and amount of granulation of the epidermal and dermal layers. To find migrated SPION-labeled MSCs in the wound, the sectioned tissue was stained with Prussian blue.
Coculture of MSCs and hDFs. hDFs were cocultured with MSCs in transwells with $0.4 \mu \mathrm{m}$ pores (Corning-Costar, New York, NY, USA), which allowed for paracrine interactions without direct cell contact. For all studies, hDFs were seeded at $0.5 \times 10^{4}$ cells $/ \mathrm{cm}^{2}$ and maintained in DMEM containing $10 \%$ FBS for 3 days prior to coculture (10). MSCs were seeded at $0.5 \times 10^{4}$ cells $/ \mathrm{cm}^{2}$ in the inserts and maintained in DMEM containing 10\% FBS for 3 days prior to coculture, which allowed the MSCs to reach 70-80\% confluence. MSC-containing inserts were then added to the dermal fibroblast wells and flooded with fresh medium containing $2 \%$ FBS. Controls consisted of dermal fibroblasts cocultured with an empty insert.

Cell proliferation assay. To verify the effect of MSCs on hDF proliferation, hDFs were cocultured with MSCs in transwells with $0.4 \mu \mathrm{m}$ pores (Corning-Costar). The hDFs were seeded at $0.5 \times 10^{4}$ cells $/ \mathrm{cm}^{2}$ in 24-well plates and maintained in DMEM with $10 \%$ FBS for 3 days prior to coculture. MSCs were seeded at $0.5 \times 10^{4} \mathrm{cells} / \mathrm{cm}^{2}$ in the inserts and maintained in the same medium for 3 days prior to coculture, which allowed the MSCs to reach $70-80 \%$ confluence $(10,15)$. MSC-containing inserts were then added to the hDF wells and flooded with fresh media containing 2\% FBS. Controls consisted of hDFs cocultured with an empty insert. At 24, 48 and $72 \mathrm{~h}$, hDF proliferation was measured using a tetrazolium reagent, 2-(4-indophenyl)-3-(4-nitrophenyl)-5-(2,4-disulphophenyl)2H-tetrazolium monosodium salt (WST-1, Cell Counting kit; Beyotime, Beijing, China). hDFs were incubated with WST-1 for $4 \mathrm{~h}$ at $37^{\circ} \mathrm{C}$ (27-30). The staining intensity in the medium was measured by determining the absorbance at $450 \mathrm{~nm}$, and the data were expressed as ratios of the control value. The data are from three independent experiments performed in duplicate.

Scratch wound closure assay. We analyzed the effect of MSCs on dermal fibroblast migration using a scratch wound assay. MSCs were seeded at $0.5 \times 10^{4}$ cells $/ \mathrm{cm}^{2}$ in the inserts ( $0.4 \mu \mathrm{m}$ pores) and maintained in the same medium for 3 days prior to coculture, which allowed the MSCs to reach 70-80\% confluence $(10,15)$. hDFs were seeded at $0.5 \times 10^{4} \mathrm{cells} / \mathrm{cm}^{2}$ in 6-well plates and maintained in DMEM containing 10\% FBS and maintained until reaching 80-90\% confluence (Day 3 post-seeding). Confluent hDFs were then scored with a sterile pipette tip to leave a scratch of $\sim 0.4-0.5 \mathrm{~mm}$ in width. Each well was washed twice with PBS to remove the cell debris and then either an empty control insert or an MSC-containing insert was placed in the scratched hDF well with DMEM containing $2 \%$ FBS. Cell migration into the scratch was photographed at $0,24,48$ and $72 \mathrm{~h}$ using an inverted microscope. The results are presented as the percentage of wound healing, which was calculated as follows: [Wound Area (initial) - Wound Area(final)]/Wound area (initial) x100 $(10,15)$. The data are from three independent experiments performed in duplicate.

Transwell migration assay. We also measured hDF migration using a transwell migration assay $(8.0 \mu \mathrm{m}$ pore size; CorningCostar) according to the manufacturer's protocol. In brief, MSCs were seeded in the bottom chamber of 24-well plates at $0.5 \times 10^{4}$ cells $/ \mathrm{cm}^{2}$; control bottom chambers contained medium 
Table I. Primers for real-time PCR analysis.

\begin{tabular}{lll}
\hline Gene & \multicolumn{1}{c}{ Forward primer } & \multicolumn{1}{c}{ Reverse primer } \\
\hline VEGF & TCACAGGTACAGGGATGAGGACAC & CAAAGCACAGCAATGTCCTGAAG \\
KGF & CACAGTGGTACCTGAGGATCGATAA & GCCACTGTCCTGATTTCCATGATA \\
TGF- $\beta$ & GCGACTCGCCAGAGTGGTTA & GTTGATGTCCACTTGCAGTGTGTTA \\
bFGF & GTGTGCTAACCGTTACCTGGCTATG & CCAGTTCGTTTCAGTGCCACA \\
Collagen I & CCCGGGTTTCAGAGACAACTTC & TCCACATGCTTTATTCCAGCAATC \\
GAPDH & GAAGGTGAAGGTCGGAGTC & GAAGATGGTGATGGGATTTC \\
\hline
\end{tabular}

VEGF, vascular endothelial growth factor; KGF, keratinocyte growth factor; TGF- $\beta$, transforming growth factor- $\beta$; bFGF, basic fibroblast growth factor; GAPDH, glyceraldehyde-3-phosphate-dehydrogenase.

without cells. A cell culture insert with $8.0 \mu \mathrm{m}$ pores was placed in the well, and $\mathrm{hDF}$ at $0.5 \times 10^{4}$ cells $/ \mathrm{cm}^{2}$ was added to the insert and incubated at $37^{\circ} \mathrm{C}$ in $5 \% \mathrm{CO}_{2}$ for 12,24 or $48 \mathrm{~h}$. Following incubation, the cells were removed from the upper chamber, and the cells on the bottom side of the insert (migrated cells) were fixed with paraformaldehyde (PFA), stained with hematoxylin and photographed. Cells were counted from eight randomly selected regions per well. The data are from three independent experiments performed in duplicate.

Real-time PCR analysis. Total RNA was extracted from hDF $48 \mathrm{~h}$ after the initiation of coculture with MSCs using TRIzol Reagent (Invitrogen). The concentration of extracted RNA was determined using an ultraviolet spectrophotometer. cDNA was synthesized from $500 \mathrm{ng}$ of total RNA using PrimeScript ${ }^{\mathrm{TM}}$ RT reagent (Takara Bio, Inc.). The primers used for real-time PCR are shown in Table I. The reactions were performed using the SYBR PrimeScript RT-PCR kit (Takara Bio, Inc.) with an ABI 7500 Sequence Detection System (Applied Biosystems). Each experiment was performed with three biological replicates. As an internal control, levels of glyceraldehyde3 -phosphate-dehydrogenase (GAPDH) were quantified in parallel with the target genes. Normalization and fold changes were calculated using the $\Delta \Delta \mathrm{Ct}$ method.

Western blotting of type I collagen expressed by hDFs. The $\mathrm{hDFs}$ were washed three times with ice-cold PBS and prepared with RIPA buffer (50 mM Tris-HCl, $0.5 \%$ deoxycholate, $1 \%$ Nonidet P-40, $150 \mathrm{mM} \mathrm{NaCl}$ and $0.1 \%$ SDS) containing a protease inhibitor mixture (Roche Diagnostics) $48 \mathrm{~h}$ after the initiation of coculture with MSCs. The samples were separated by SDS-PAGE and then transferred to a polyvinylidene difluoride membrane (Millipore, Bedford, MA, USA). The membrane was blocked with $5 \%$ nonfat milk and incubated with a type I collagen primary antibody ( $\mathrm{R} \& \mathrm{D}$, USA) at $4^{\circ} \mathrm{C}$ overnight. Then the blots were incubated with an HRP-conjugated secondary $\mathrm{Ab}$ (Proteintech, USA) for $1 \mathrm{~h}$ at room temperature. Immunoreactive bands were visualized by the SuperSignal West Pico Chemiluminescent Substrate (Pierce, Rockford, IL,USA) using LAS-3000 mini (Fujifilm).

Statistical analysis. All experiments were repeated at least three times. The data are shown as the means \pm SD. Comparisons between groups were analyzed using a t-test. All the statistical analyses were preformed using SPSS 19.0 computer software. P-values $<0.05$ were considered to indicate statistically significant differences.

\section{Results}

Characterization of MSCs from different tissues. MSCs were isolated from human amnion, bone marrow and adipose tissue. They were expanded on a plastic dish and exhibited similar fibroblast-like morphologies (Fig. 1A). Stem cell-related cell surface markers were assessed using flow cytometry to characterize isolated MSCs. All types of MSCs expressed CD105 and CD90, but not CD34, CD14, CD45 or HLA-DR, consistent with previous reports (15,31) (Fig. 1B). MSCs were further characterized by confirming their ability to undergo specific osteogenic, chondrogenic and adipogenic differentiation, as examined by alkaline phosphatase staining, Alcian blue staining and Oil Red O staining (Fig. 1A), respectively. Therefore, these MSC populations have the ability to differentiate into osteocytes, chondrocytes and adipocytes.

Comparison of hAMSCs, hBMSCs and hADSCs for wound healing in vivo. To compare the roles of hAMSCs, hBMSCs and hADSCs in wound healing $(15,26)$, we injected them into cutaneous wounds on mice. The MSC-injected groups exhibited accelerated wound closure compared with the controls. The enhancement appeared early, at 3 days after transplantation, and became more evident after 7 days (Fig. 2A). Quantitative analysis demonstrated that an hADSC injection had the most pronounced effect on wound closure, followed by the hBMSCs, hAMSCs and the control groups at 3, 7, 10 and 14 days after transplantation (Fig. 2B). Although a slight improvement in wound healing was noted in the hAMSC and hBMSC groups, there was no statistically significant difference between the two groups. Histological evaluation of the wounds at 7, 14 and 21 days after surgery showed enhanced re-epithelialization in the hADSC group compared with all other groups (Fig. 2C). In addition, granulation tissue in the hADSC-injected group appeared to be thicker and larger. These results indicated that hADSCs had an evident effect on wound healing compared with hAMSCs or hBMSCs. To find migrated MSCs in the wound skin, we labeled MSCs with SPIONs and stained the slide-cultured cells and sectioned tissue by Prussian blue staining (Fig. 2D). 
A

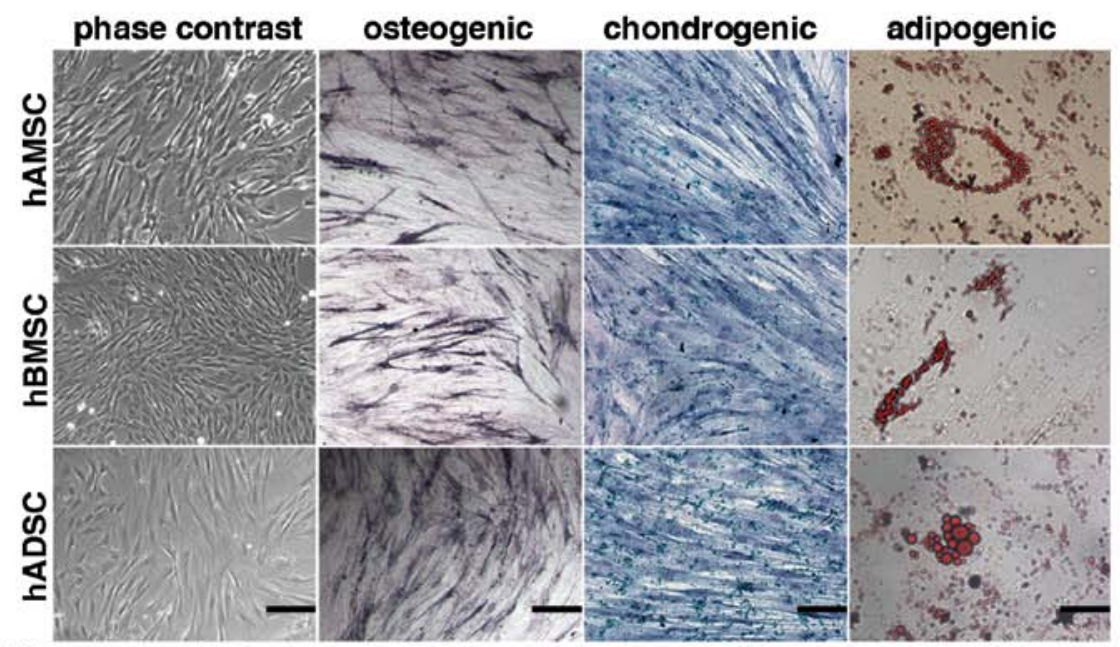

B
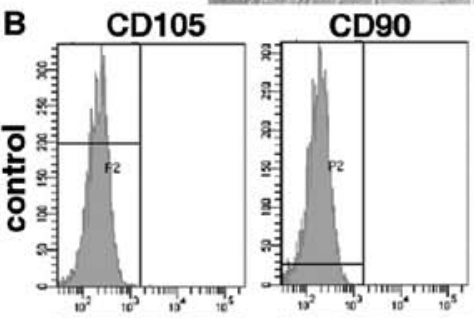

CD45
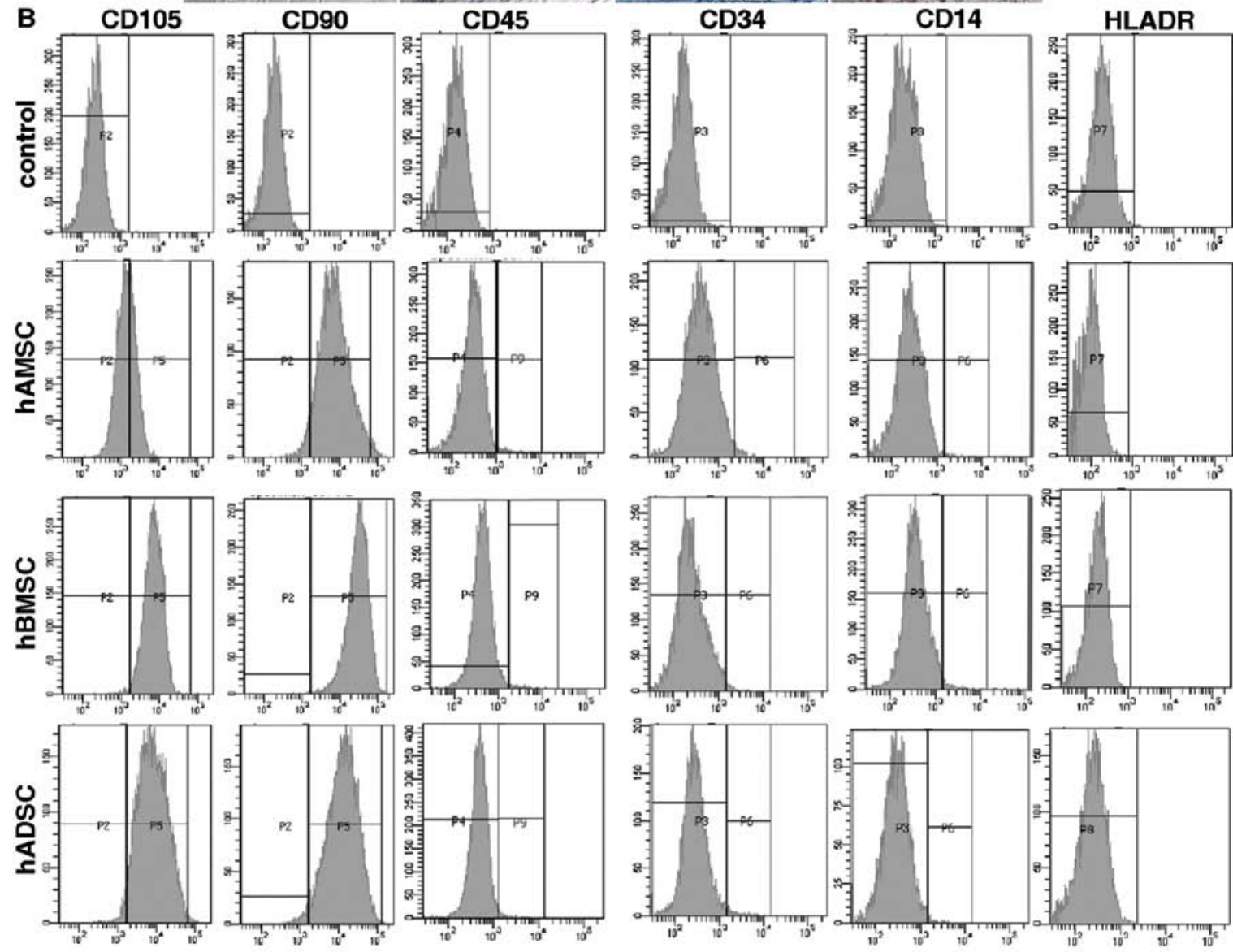

Figure 1. Characterization of hAMSCs, hBMSCs and hADSCs. (A) Morphologic comparison of hAMSCs, hBMSCs and hADSCs at passage 3. All cells were similarly adherent to plastic and had a spindle-shaped morphology, but hAMSCs adhered slightly longer than hBMSCs and hADSCs. Functional assays for the differentiation of cultured MSCs in all of our MSC populations. Osteogenic, chondrogenic and adipogenic differentiation could be induced under the appropriate conditions, as indicated by alkaline phosphatase staining, Alcian blue staining or Oil Red O staining. Scale bar is equivalent to $50 \mu \mathrm{m}$. (B) Passage $3 \mathrm{hAMSCs}$ hBMSCs and hADSCs were labeled with antibodies against the indicated antigens and analyzed by flow cytometry to obtain representative immunophenotypes.

Mechanisms of woundhealing differ among hAMSCs, hBMSCs and $h A D S C s$. Since the proliferation of hDFs is an important aspect of wound healing $(10,32)$, we compared the differences in the stimulatory effects of hAMSCs, hBMSCs and hADSCs on hDF proliferation in transwell coculture experiments to clarify how these cell types altered hDF proliferation. WST analysis of hDFs revealed that hDFs cultured in transwells with an MSC insert showed enhanced proliferation compared with control hDFs cultured with an empty insert after 48 and
$72 \mathrm{~h}$ of coculture. However, there was no significant difference in fibroblast proliferation between the hAMSC, hBMSC and hADSC inserts (Fig. 3A).

To examine whether hADSCs exhibited biological effects relevant to hDF migration $(10,32)$, we compared the effects of hAMSCs, hBMSCs and hADSCs on hDF migration. Images of scratch wounds on hDF monolayers were captured 24, 48 and $72 \mathrm{~h}$ after scratching to show that hDF migration into the scratch wound area was accelerated in coculture with hADSCs 
A

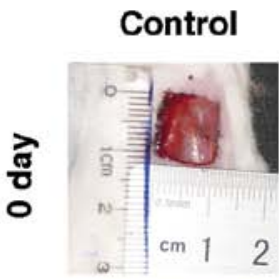

hAMSC

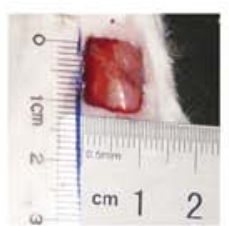

$\sum_{m}^{1}$
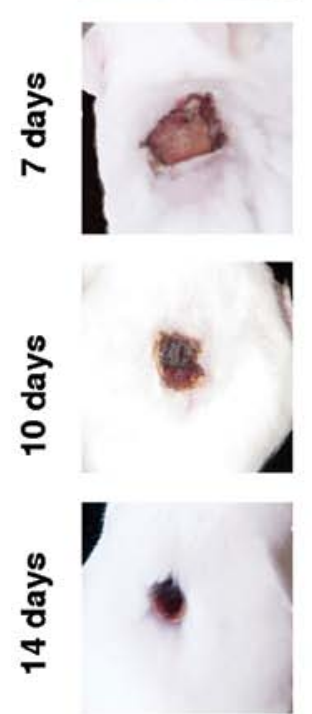
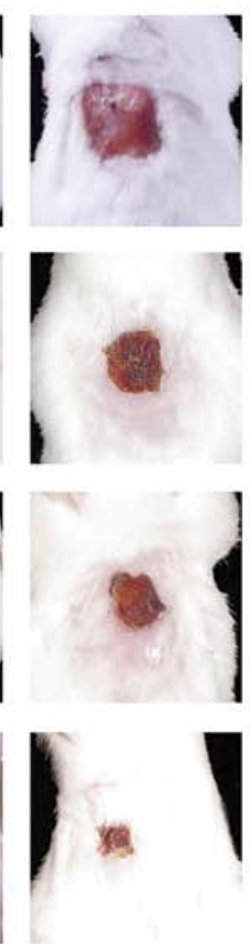

1
hBMSC

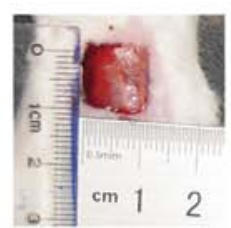

hADSC
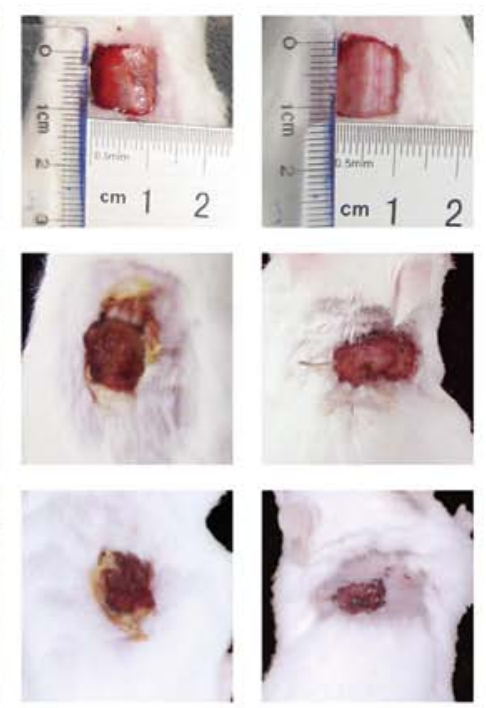

(1)

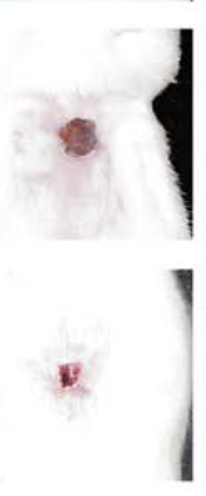

B
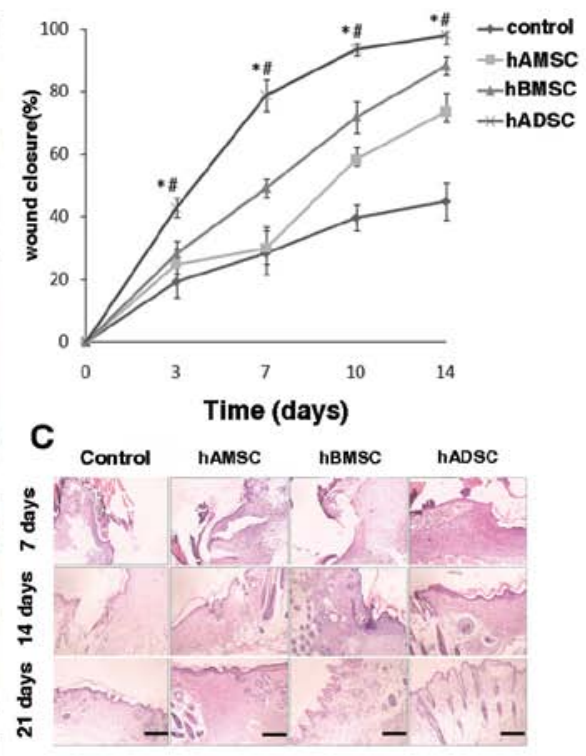

D

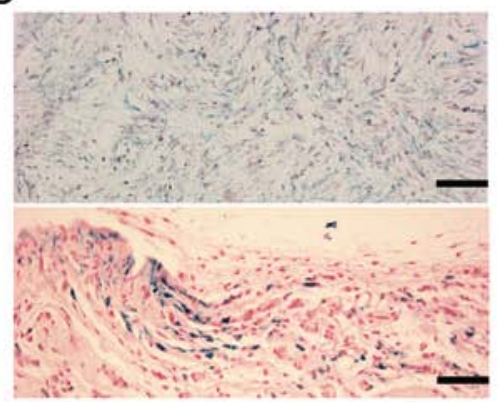

Figure 2. Wound healing effects of hAMSCs, hBMSCs and hADSCs in a mouse model of cutaneous wounds. (A) Comparison of wound closure among the four groups. Representative photographs of the wounds at Days 0, 3, 7, 10 and 14 after grafting. (B) Quantitative analysis demonstrated that wound size was reduced in the MSC-injected groups compared with the control after 3 days. The hADSC-injected group demonstrated significant improvement in wound healing compared with the hAMSC- and hBMSC-injected groups. Data represent the means $\pm \mathrm{SD}(\mathrm{n}=3)$. " $\mathrm{P}<0.05$, significantly different from the control group; " $\mathrm{P}<0.05$, significantly different from the hAMSC and hBMSC groups. (C) Histological analysis of repaired skin, which was stained with $\mathrm{H} \& \mathrm{E}, 7,14$ and 21 days after surgery. Comparison of sections stained with H\&E among the four groups 7, 14 and 21 days after grafting. The hADSC group had a thin epidermis with mature differentiation and collagen bundles deposited with recovered skin appendages in the dermal layer. (D) To find migrated MSCs in the wound skin, we labeled MSCs with SPIONs and stained the slide-cultured cells and sectioned tissue by Prussian blue staining. Scale bar is equivalent to $50 \mu \mathrm{m}$.

compared with other cocultures (Fig. 3B). Quantitative analysis confirmed that hADSCs were superior to hAMSCs and hBMSCs in promoting hDF migration (Fig. 3C).

In transwell cell migration assays, hAMSCs, hBMSCs or hADSCs were seeded in the bottom chamber of a transwell and hDFs were loaded into the top chamber. hDFs migrated into the bottom chamber and were stained 12, 24 and $48 \mathrm{~h}$ after initiating coculture (Fig. 3D). Quantitative analysis confirmed that hDF migration into the bottom chamber was more accelerated in the presence of hADSCs (Fig. 3E).

Role of cytokines and ECM in the wound healing action of $h A D S C s$. To understand how the molecular mechanisms of hADSCs improved wound healing, we compared the expression of wound-healing-related cytokines $(25,26,32)$ and ECM proteins (15) in hDFs cocultured with hAMSCs, hBMSCs or hADSCs. Real-time PCR was performed for the cytokine vascular endothelial growth factor (VEGF), basic fibroblast growth factor (bFGF), keratinocyte growth factor (KGF) and TGF- $\beta$, which confirmed significantly higher mRNA expres- sion of these cytokine genes in hDFs cocultured for $48 \mathrm{~h}$ with hADSCs than with other MSCs (Fig. 4A).

Next, we investigated the effects of hADSCs on hDF expression of ECM type I collagen, as measured by mRNA and protein levels. Real-time PCR analysis (Fig. 4B) showed that hADSCs have a more potent effect on type I collagen expression by hDFs than either hAMSCs or hBMSCs. Western blotting (Fig. 4C) showed that hADSCs also have a more potent effect on type I collagen expression by hDFs. These results indicate that hDFs cocultured with hADSCs for $48 \mathrm{~h}$ have significantly upregulated expression of type I collagen, as measured by mRNA and protein levels.

\section{Discussion}

Stem cell therapy is a promising approach for treating cutaneous wounds. Embryonic stem cells (ESCs) have a great capacity for self-renewal and plasticity, but their use is limited by political, scientific, and ethical considerations. The use of adult stem cells, particularly mesenchymal stem cells (MSCs), 


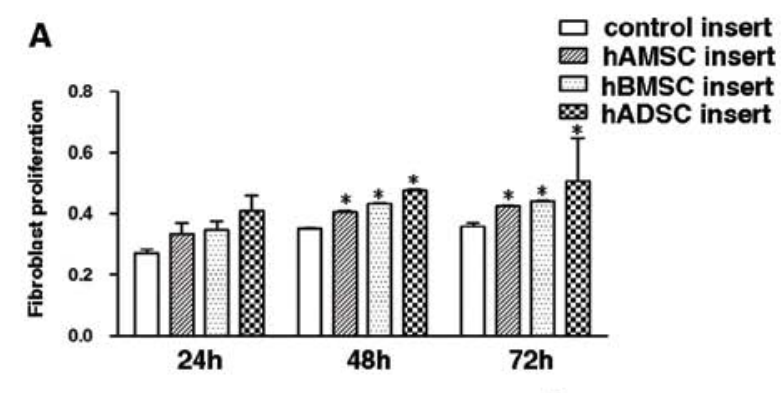

B

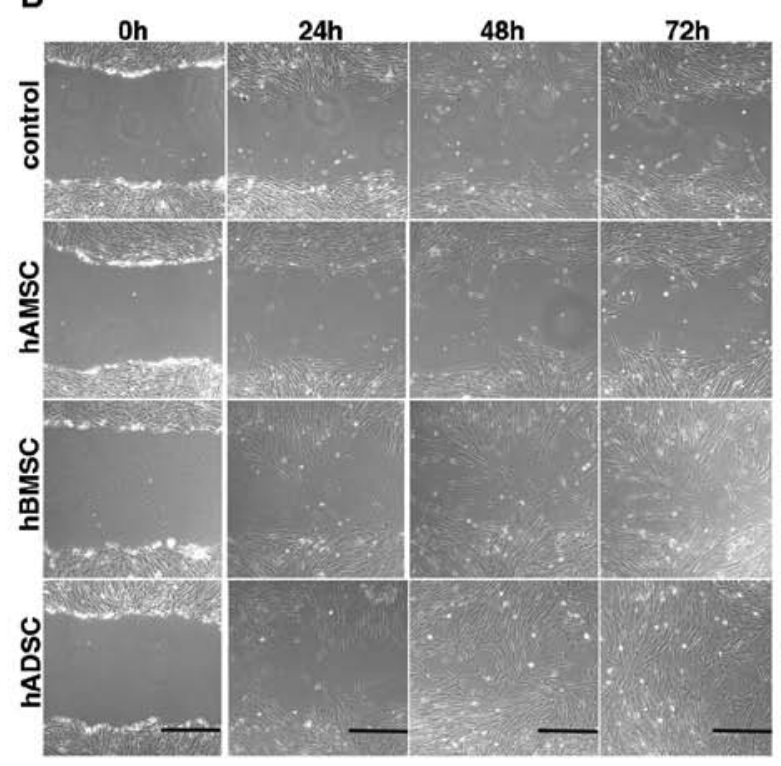

C

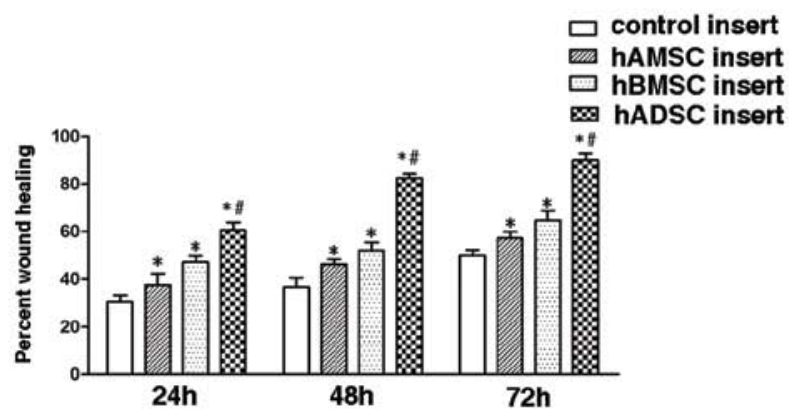

D

E
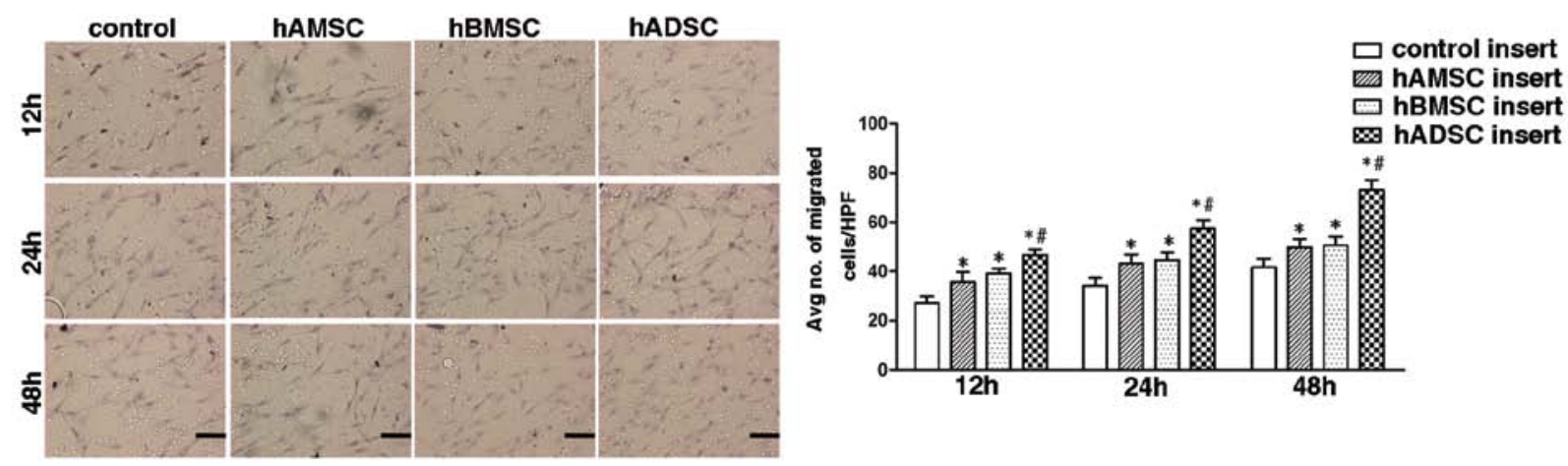

Figure 3. Effects of MSCs on the proliferation and migration of hDFs. (A) hDFs were cocultured with an insert containing hAMSCs, hBMSCs or hADSCs or an empty insert (control group). At 24, 48 and $72 \mathrm{~h}$ after beginning the coculture, the proliferation of dermal fibroblasts was measured with a WST-1 assay. $\mathrm{hDFs}$ cultured in transwells with MSC inserts showed enhanced proliferation compared with control hDFs cultured with an empty insert $48 \mathrm{~h}(\mathrm{P}<0.05)$ and $72 \mathrm{~h}(\mathrm{P}<0.05)$ after starting the coculture. However, there was no significant difference in fibroblast proliferation between the hAMSC, hBMSC and hADSC inserts. (B) Migration of hDFs into the scratch wound after 24, 48 and $72 \mathrm{~h}$ of coculture with hAMSCs, hBMSCs or hADSCs in scratch wound assay. (C) Rate of movement after 24,48 and $72 \mathrm{~h}$ of coculture. The rate of movement was significantly greater for hDFs cocultured with hADSCs compared with hAMSCs and hBMSCs. (D) Photographs of hematoxylin stained membranes in transwell hDF migration assays after 12, 24 and $48 \mathrm{~h}$ of coculture with hAMSCs, hBMSCs or hADSCs. (E) Rate of movement after 12, 24 and $48 \mathrm{~h}$ of coculture. Results indicated that hDF migration into the bottom chamber was accelerated in the presence of hADSCs compared with the others. Data represent the means $\pm \mathrm{SD}(\mathrm{n}=3)$. ${ }^{*} \mathrm{P}<0.05$, significantly different from the control group; ${ }^{\sharp} \mathrm{P}<0.05$, significantly different from the hAMSC and hBMSC groups. Scale bar is equivalent to $50 \mu \mathrm{m}$.

is not burdened by many of these problems. Marrow stromal cells are adult stem cells with unique immunologic tolerance, which allows for their engraftment into a xenogeneic environment while preserving their ability to be recruited to an injured tissue (10-14).

Dermal fibroblast responses to injury are essential for cutaneous wound repair. The initial responses of fibroblasts adjacent to the wound site and subsequent migration into the wound are due to their essential role in wound contraction, extracellular matrix (ECM) deposition and tissue remodeling $(10,33,34)$. In fact, earlier studies have shown that MSCs may provide an important early signal for dermal fibroblast responses to cutaneous injury $(10,15)$. MSCs are present in various tissues including amnion, bone marrow and adipose tissues; however, hADSCs are a more readily available autologous stem cell source than the others. It remains unclear 

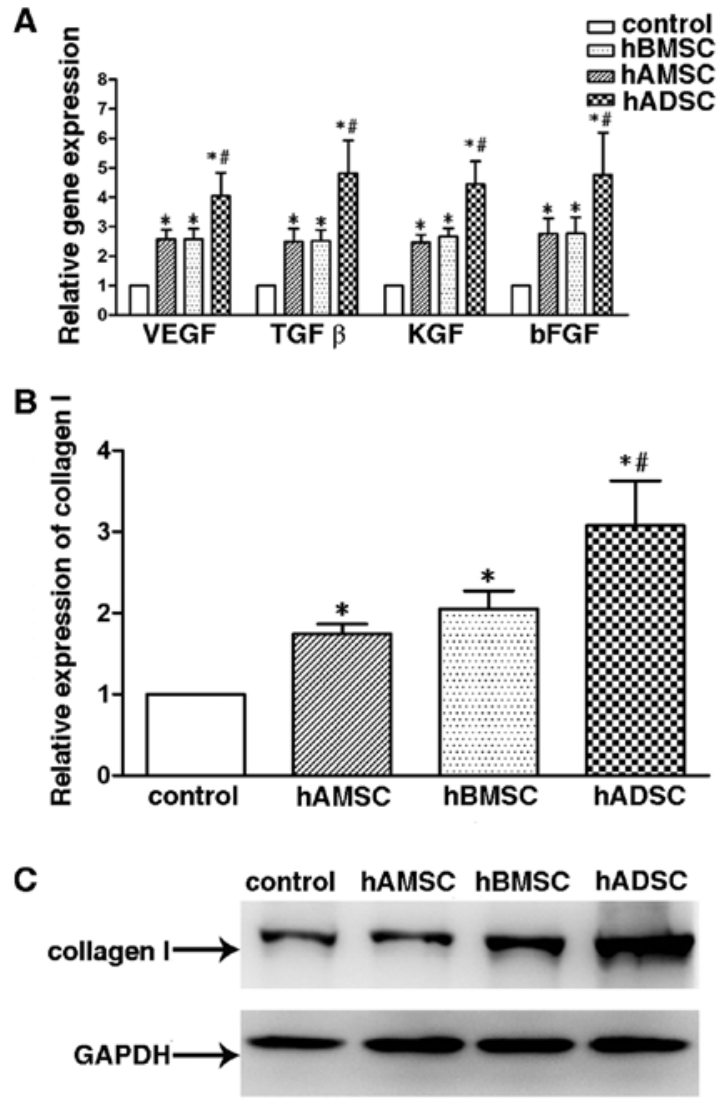

Figure 4. (A) Real-time quantitative RT-PCR analysis of hDF expression of VEGF, bFGF, KGF and TGF- $\beta$ after coculture with MSCs for $48 \mathrm{~h}$ Results indicated that hDFs cocultured with hADSCs for $48 \mathrm{~h}$ significantly upregulated RNA expression of VEGF, bFGF, KGF and TGF- $\beta$. (B and C) Effects of MSCs on the expression of type I collagen by hDFs. Expression of type I collagen was determined by (B) real-time PCR and (C) western blot analyses. The hADSC group increased type I collagen expression more than the hAMSC and hBMSC groups, as determined by the mRNA and protein levels. Data represent the means $\pm \mathrm{SD}(\mathrm{n}=3) .{ }^{*} \mathrm{P}<0.05$, significantly different from the control group; ${ }^{*} \mathrm{P}<0.05$, significantly different from the hAMSC and hBMSC groups.

whether the wound healing potency of hADSCs is comparable or superior to that of hAMSCs and hBMSCs.

In this study, we cultured MSCs from human amnion tissue, bone marrow and adipose tissue under standard conditions and evaluated them according to the following characteristics: ability for self-renewal, expansion in cell culture, expression of stem-cell-related markers and the capacity to differentiate into osteoblasts, chondrocytes and adipocytes. Then, we used SPIONs to label MSCs to monitor their fate by Prussian blue staining (19,22-24). In a mouse model of excisional wound healing, we injected equal numbers of labeled hAMSCs, hBMSCs or hADSCs around the wound to compare the therapeutic effects of the three types of cells. HAM has been used as a dressing for surface wounds based on its anti-inflammatory and antimicrobial effects (35-40). Our studies revealed that hADSCs significantly reduced the wound size and accelerated re-epithelialization from the wound edge, suggesting that hADSC transplantation is a more potent therapeutic treatment for skin wounds than hBMSC or hAMSC transplantation.

The underlying mechanisms responsible for the superiority of hADSCs in therapeutic wound healing remain unknown. Current studies have suggested that MSCs may promote tissue repair by secreting paracrine signals that improve injury resolution by resident skin cells, including dermal fibroblasts, keratinocytes and endothelial cells $(10,26)$. In this study, we used transwell experiments to coculture dermal fibroblasts with hAMSCs, hBMSCs and hADSCs. In this coculture model, hADSCs were superior to the others in promoting $\mathrm{hDF}$ migration in a scratch wound assay and transwell migration assay. To understand the molecular mechanisms of hADSCinduced wound healing, we compared the expression of wound-healing-related cytokines and ECM proteins in hDFs cocultured with hAMSCs, hBMSCs or hADSCs. Real-time PCR showed that VEGF, bFGF, KGF and TGF- $\beta$ expression were expressed more highly in hDFs cocultured with hADSCs than in those cocultured with either hBMSCs or hAMSCs. Collagen I expression was also higher in hDFs cocultured with hADSCs than with hBMSCs or hAMSCs, as measured by real-time PCR and western blotting. However, we did not observe significant differences in dermal fibroblast proliferation between the different groups.

Fibroblasts are the major stromal cells in the dermis and several other tissues. They are known to appear during the wound healing process and release numerous cytokines that lead to wound healing $(10,15)$. Previous studies of wound healing have shown that the deposition of collagen and the expression of key growth factors, such as bFGF, VEGF, KGF and TGF- $\beta$, are important in wound healing $(25,26,32,41)$. Basic FGF is an angiogenic growth factor that augments the production of ECM components, fibroblast proliferation and migration for angiogenesis, and re-epithelialization, and it is an efficient mediator of VEGF activity (42-47). Keratinocyte growth factor, another member of the FGF family, promotes re-epithelialization and stimulates granulation tissue formation and collagen deposition in the healing wound. VEGF, which is a family of platelet-derived growth factors, induces angiogenesis and endothelial cell proliferation and also stimulates epithelialization, collagen deposition and vascular permeability (48). TGF- $\beta$ is a potent stimulator of fibroblast proliferation and collagen synthesis. Early experiments on TGF- $\beta$ suggested that this factor was involved in accelerating re-epithelialization and enhancing wound healing (42). In this study, we demonstrated that hADSCs promoted the expression of VEGF, bFGF, KGF and TGF- $\beta$ and the expression of collagen I by hDFs and also promoted hDF migration. Although the soluble factors released by hADSCs that are responsible for the increased wound healing characteristics of $\mathrm{hDFs}$ remain to be identified, hADSCs may still be a superior cell treatment as a therapeutic for wound healing.

It is difficult to obtain sufficient hBMSCs for clinical trials in some patients; however, hADSCs can easily be isolated from a small amount of adipose tissue and rapidly expanded in culture. The data in this study demonstrated that hADSCs show better wound repair activity than hBMSCs and hAMSCs. Therefore, hADSC transplantation may be one of the most attractive cell therapies for the treatment of wound repair.

\section{Acknowledgements}

This study was supported by the National Basic Science and Development Program (973 Program 2012CB518103) and the Shenyang Science and Technology Program (nos. 090063, F10-222-4-00 and F11-262-9-01). 


\section{References}

1. Chen M, Przyborowski M and Berthiaume F: Stem cells for skin tissue engineering and wound healing. Crit Rev Biomed Eng 37: 399-421, 2009.

2. Walter MN, Wright KT, Fuller HR, MacNeil S and Johnson WE: Mesenchymal stem cell-conditioned medium accelerates skin wound healing: an in vitro study of fibroblast and keratinocyte scratch assays. Exp Cell Res 316: 1271-1281, 2010.

3. Han SK, Yoon TH, Lee DG, Lee MA and Kim WK: Potential of human bone marrow stromal cells to accelerate wound healing in vitro. Ann Plast Surg 55: 414-419, 2005.

4. Kim Y,Kim H, Cho H, Bae Y, Suh K and Jung J: Direct comparison of human mesenchymal stem cells derived from adipose tissues and bone marrow in mediating neovascularization in response to vascular ischemia. Cell Physiol Biochem 20: 867-876, 2007.

5. Prockop DJ: Marrow stromal cells as stem cells for nonhematopoietic tissues. Science 276: 71-74, 1997.

6. Pittenger MF, Mackay AM, Beck SC, et al: Multilineage potential of adult human mesenchymal stem cells. Science 284 143-147, 1999.

7. Zuk PA, Zhu M, Mizuno H, et al: Multilineage cells from human adipose tissue: implications for cell-based therapies. Tissue Eng 7: 211-228, 2001

8. Safford KM, Hicok KC, Safford SD, et al: Neurogenic differentiation of murine and human adipose-derived stromal cells Biochem Biophys Res Commun 294: 371-379, 2002.

9. Reyes M, Dudek A, Jahagirdar B, Koodie L, Marker PH and Verfaillie CM: Origin of endothelial progenitors in human postnatal bone marrow. J Clin Invest 109: 337-346, 2002.

10. Smith AN, Willis E, Chan VT, Muffley LA, Isik FF, Gibran NS and Hocking AM: Mesenchymal stem cells induce dermal fibroblast responses to injury. Exp Cell Res 316: 48-54, 2010.

11. Xing L, Franz MG, Marcelo CL, Smith CA, Marshall VS and Robson MC: Amnion-derived multipotent progenitor cells increase gain of incisional breaking strength and decrease incidence and severity of acute wound failure. J Burns Wounds 7: e5, 2007.

12. Kim SS, Song CK, Shon SK, Lee KY, Kim CH, Lee MJ and Wang L: Effects of human amniotic membrane grafts combined with marrow mesenchymal stem cells on healing of full-thickness skin defects in rabbits. Cell Tissue Res 336: 59-66, 2009.

13. Jung KH, Song SU, Yi T, et al: Human bone marrow-derived clonal mesenchymal stem cells inhibit inflammation and reduce acute pancreatitis in rats. Gastroenterology 140: 998-1008, 2011.

14. Saito T, Kuang JQ, Bittira B, Al-Khaldi A and Chiu RC: Xenotransplant cardiac chimera: immune tolerance of adult stem cells. Ann Thorac Surg 74: 19-24, 2002.

15. Kim WS, Park BS, Sung JH, et al: Wound healing effect of adipose-derived stem cells: a critical role of secretory factors on human dermal fibroblasts. J Dermatol Sci 48: 15-24, 2007.

16. Falanga V, Iwamoto $\mathrm{S}$, Chartier M, et al: Autologous bone marrow-derived cultured mesenchymal stem cells delivered in a fibrin spray accelerate healing in murine and human cutaneous wounds. Tissue Eng 13: 1299-1312, 2007.

17. Javazon EH, Keswani SG, Badillo AT, et al: Enhanced epithelia gap closure and increased angiogenesis in wounds of diabetic mice treated with adult murine bone marrow stromal progenitor cells. Wound Repair Regen 15: 350-359, 2007.

18. Lin CD, Allori AC, Macklin JE, et al: Topical lineage-negative progenitor-cell therapy for diabetic wounds. Plast Reconstr Surg 122: 1341-1351, 2008.

19. Wu Y, Chen L, Scott PG and Tredget EE: Mesenchymal stem cells enhance wound healing through differentiation and angiogenesis. Stem Cells 25: 2648-2659, 2007

20. Wu Y, Wang JF, Scott PG and Tredget EE: Bone marrow-derived stem cells in wound healing: a review. Wound Repair Regen 15 (Suppl 1): S18-S26, 2007.

21. Sasaki M, Abe R, Fujita Y, Ando S, Inokuma D and Shimizu H: Mesenchymal stem cells are recruited into wounded skin and contribute to wound repair by transdifferentiation into multiple skin cell type. J Immunol 180: 2581-2587, 2008.

22. Yoo JH, Park C, Jung DI, et al: In vivo cell tracking of canine allogenic mesenchymal stem cells administration via renal arterial catheterization and physiopathological effects on the kidney in two healthy dogs. J Vet Med Sci 73: 269-274, 2011.

23. Balakumaran A, Pawelczyk E, Ren J, et al: Superparamagnetic iron oxide nanoparticles labeling of bone marrow stromal (mesenchymal) cells does not affect their 'stemness'. PLoS One 5 e11462, 2010
24. Chen L, Tredget EE, Wu PY and Wu Y: Paracrine factors of mesenchymal stem cells recruit macrophages and endothelial lineage cells and enhance wound healing. PLoS One 3: e1886, 2008.

25. Yu M, Cai L, Liang M, et al: Alteration of NRSF expression exacerbating 1-methyl-4-phenyl-pyridinium ion-induced cell death of SH-SY5Y cells. Neurosci Res 65: 236-244, 2009.

26. Li H, Wang L, Ye L, et al: Influence of Pseudomonas aeruginosa quorum sensing signal molecule $\mathrm{N}$-(3-oxododecanoyl) homoserine lactone on mast cells. Med Microbiol Immunol 198 : 113-121, 2009.

27. Cheng Y, Hu R, Jin $\mathrm{H}$, et al: Effect of 14-3-3 tau protein on differentiation in BeWo choriocarcinoma cells. Placenta 31: 60-66, 2010.

28. Liu S, Yang P, Kang H, et al: NDRG2 induced by oxidized LDL in macrophages antagonizes growth factor productions via selectively inhibiting ERK activation. Biochim Biophys Acta 1801: 106-113, 2010.

29. Wagner W, Wein F, Seckinger A, et al: Comparative characteristics of mesenchymal stem cells from human bone marrow, adipose tissue, and umbilical cord blood. Exp Hematol 33: 1402-1416, 2005.

30. Hartlapp I, Abe R, Saeed RW, et al: Fibrocytes induce an angiogenic phenotype in cultured endothelial cells and promote angiogenesis in vivo. FASEB J 15: 2215-2224, 2001

31. Martin P: Wound healing-aiming for perfect skin regeneration. Science 276: 75-81, 1997 .

32. Singer AJ and Clark RA: Cutaneous wound healing. N Engl J Med 341: 738-746, 1999.

33. Shojaku H, Takakura H, Okabe M, Fujisaka M, Watanabe $Y$ and Nikaido T: Effect of hyperdry amniotic membrane patches attached over the bony surface of mastoid cavities in canal wall down tympanoplasty. Laryngoscope 121: 1953-1957, 2011.

34. Gruss JS and Jirsch DW: Human amniotic membrane: a versatile wound dressing. Can Med Assoc J 118: 1237-1246, 1978.

35. Krishnamurithy G, Shilpa PN, Ahmad RE, Sulaiman S, Ng CL and Kamarul T: Human amniotic membrane as a chondrocyte carrier vehicle/substrate: in vitro study. J Biomed Mater Res A 99: 500-506, 2011.

36. McGhee CN and Patel DV: Mooren's ulcer and amniotic membrane transplant: a simple surgical solution? Clin Experiment Ophthalmol 39: 383-385, 2011

37. Pessolato AG, Martins Ddos S, Ambrósio CE, Mançanares CA and de Carvalho AF: Propolis and amnion reepithelialise second-degree burns in rats. Burns 37: 1192-1201, 2011.

38. Toda A, Okabe M, Yoshida T and Nikaido T: The potential of amniotic membrane/amnion-derived cells for regeneration of various tissues. J Pharmacol Sci 105: 215-228, 2007.

39. Chesney J, Metz C, Stavitsky AB, Bacher M and Bucala R Regulated production of type I collagen and inflammatory cytokines by peripheral blood fibrocytes. J Immunol 160: 419-425, 1998.

40. Belgore F, Lip GY and Blann AD: Basic fibroblast growth factor induces the secretion of vascular endothelial growth factor by human aortic smooth muscle cells but not by endothelial cells Eur J Clin Invest 33: 833-839, 2003.

41. Zhang F, Oswald T, Lin S, et al: Vascular endothelial growth factor (VEGF) expression and the effect of exogenous VEGF on survival of a random flap in the rat. Br J Plast Surg 56: 653-659, 2003.

42. Yang R, Thomas GR, Bunting S, et al: Effects of vascular endothelial growth factor on hemodynamics and cardiac performance. J Cardiovasc Pharmacol 27: 838-844, 1996.

43. Barrientos S, Stojadinovic O, Golinko MS, Brem H and Tomic-Canic M: Growth factors and cytokines in wound healing. Wound Repair Regen 16: 585-601, 2008.

44. Postlethwaite AE, Keski-Oja J, Moses HL and Kang AH: Stimulation of the chemotactic migration of human fibroblasts by transforming growth factor beta. J Exp Med 165: 251-256, 1987.

45. Li Y, Fan J, Chen M, Li W and Woodley DT: Transforming growth factor-alpha: a major human serum factor that promotes human keratinocyte migration. J Invest Dermatol 126: 2096-2105, 2006.

46. Arnold F and West DC: Angiogenesis in wound healing. Pharmacol Ther 52: 407-422, 1991

47. Fam NP, Verma S, Kutryk M and Stewart DJ: Clinician guide to angiogenesis. Circulation 108: 2613-2618, 2003.

48. Hansen SL, Young DM and Boudreau NJ: HoxD3 expression and collagen synthesis in diabetic fibroblasts. Wound Repair Regen 11: 474-480, 2003. 